\title{
Unsedated colonoscopy is a feasible primary screening tool for colorectal cancer
}

Screening for colorectal cancer with unsedated colonoscopy is feasible according to a new study, which reports that approximately $90 \%$ of patients tolerate this procedure well.

"Although colonoscopy is the gold standard for colorectal cancer screening, most doctors and examinees consider it

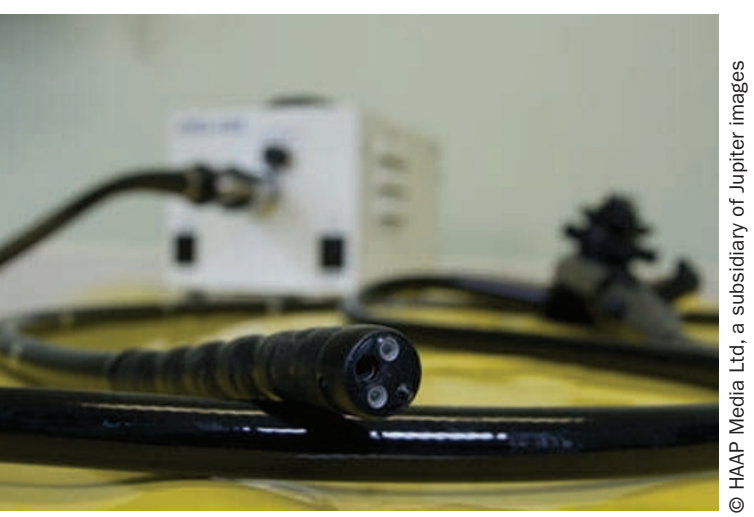

to be poorly accepted without sedation," explains Hsiu-Po Wang, lead investigator. "However, sedation is associated with an enormous cost and is not routinely available globally."

Wang and colleagues from the National Taiwan University Hospital conducted a prospective, single-center study of 261 individuals who underwent screening with unsedated colonoscopy or unsedated sigmoidoscopy. The aim of their research was to compare patients' acceptance of the two procedures in a real clinical setting, and to identify factors that could predict or improve the acceptance of unsedated colonoscopy.

There were no significant differences between the two procedures with regard to pain scores, acceptance of the procedure or patient preference for sedation; sedation was considered necessary by only $9.6 \%$ of patients in the colonoscopy group and $10.1 \%$ of patients in the sigmoidoscopy group.

"Our results indicate that unsedated colonoscopy should be recommended when sedation is not readily affordable or available," says Wang. He adds that "...future research should also evaluate unsedated colonoscopy in terms of costeffectiveness and access in settings with and without a shortage of resources." The researchers plan to conduct a randomized study to address the limitations of their current study and to provide more accurate evidence that can be used for policy making for future practice and screening.

Rachel Jones

Original article Liao, W. C. et al. A prospective evaluation of the feasibility of primary screening with unsedated colonoscopy. Gastrointest. Endosc. 70, 724-731 (2009). 